\title{
4 Urheberrecht und Informationsethik
}

Urheberrecht und Informationsethik zusammenzudenken, ist eine Herausforderung für beide - von Seiten professioneller Juristen vermutlich als Zumutung empfunden. Recht ist Recht und Ethik ist Ethik. Zu stark sind beide Bereiche voneinander getrennt. Recht reklamiert quasi das Monopol für die Regulierung so gut wie aller Lebensbereiche. Dieses Monopol der Verrechtlichung aller Lebensbereiche durch Politik und Rechtsprechung läuft Gefahr, sich von der Basis der moralischen Überzeugung, der „Gewohnheiten, Traditionen und Einstellungen“ der vom Recht Betroffenen abzulösen. Dadurch, so der schon zu Anfang zitierte (ehemalige) Richter des Obersten Gerichtshof in England, Jonathan Sumption, ${ }^{72}$ wird die Funktionsfähigkeit der Demokratie gefährdet. Zumindest, so die These hier, wird durch das Urheberrecht die Funktionsfähigkeit von Bildung und Wissenschaft gefährdet.

In der Tat wird hier die Frage nach der Priorität von Ethik gestellt. Die These der Priorität von Ethik gegenüber Recht - sanfter formuliert: der erfolgreichen Beeinflussung politischen Handelns durch moralisch begründete Leitideen - ist in der Politikwissenschaft, vor allem in den Theorien internationaler Beziehungen ${ }^{73}$, durchaus geläufig, zumal dann, wenn man „moralische Vorstellungen“ mit „Ideen“ übersetzt. ${ }^{74}$ Hier besteht der Konflikt zwischen Realpolitik und ideengeleiteter Politik. ${ }^{75}$ Ideengeleitete Politik ist, wie in 3.3 ausgeführt, wertbegründete Politik.

72 Vgl. den Bericht von Gina Thomas zu der BBV-Vorlesung von Jonathan Sumption - FAZ 12-8-2019; mit der These: „Kritik an der schleichenden Machtausdehnung der Judikative, die er [Sumption] als Bedrohung des fragilen Konstrukts der Demokratie sieht.“ - https://bit.ly/2lNIjbI.

73 Für eine Diskussion der verschiedenen ideen-basierten Ansätze, vor allem der breit ausdifferenzierte Debatte um die Rolle von Ideen bzw. die Bedeutung von moralischen Argumenten in der internationalen Politik vgl. (Hanrider 2008); vgl. (Risse-Kappen 1994) Ideas do not float freely; (Risse-Kappen/Sikkink 1999) The socialization of international human rights norms in domestic practices; vgl. (Maier 2003) Wissens- und ideenorientierte Ansätze in der Politikwissenschaft 74 Als klassisches Beispiel für die Wirksamkeit von „Ideen“ gilt die Annahme, dass nicht zuletzt durch die (anfänglich kaum beachteten) Vereinbarungen über Menschenrechte und Grundfreiheiten in der Schlussakte der zweijährigen Konferenz über Sicherheit und Zusammenarbeit in Europa (KSZE) in Helsinki von August 1975 sich immer mehr zivilgesellschaftliche Gruppierungen in der Sowjetunion bilden konnten, die das Einhalten von Grundfreiheiten wie Gedanken-, Gewissens-, Religions- oder Überzeugungsfreiheit reklamierten und zunehmend mit Erfolg auch durchsetzten. Dieses, so die These, habe das politische und gesellschaftliche System in der damaligen Sowjetunion so stark beeinflusst und verändert, dass es sich als politisches System schließlich auflöste.

75 Zur Rolle von Normen und Werten aus Sicht der Philosophie vgl. die Artikel in (Forst/Günther 2011) Die Herausbildung normativer Ordnungen; ebenso (Witschen 1998) Was verdient moralisch den Vorzug?; (Zippelius 2004) Verhaltenssteuerung durch Recht und kulturelle Leitideen.

Ә Open Access. (C) 2020 Rainer Kuhlen (c))BY This work is licensed under a Creative Commons Attribution 4.0 License. https://doi.org/10.1515/9783110693447-004 
Ausgangspunkt für die folgenden Überlegungen ist eine intuitiv einleuchtende, empirisch aber schwierig zu belegende These zum Zusammenhang von Ethik/Moral und Politik/Recht. ${ }^{76}$ Sie besagt, dass in einer längeren Perspektive die Politik, insbesondere das Recht, hier das Urheberrecht, nicht auf Akzeptanz von Seiten der von diesem Recht Betroffenen zählen kann, wenn die rechtlichen Regelungen nicht mit den ethisch begründeten normativen Erwartungen an den Umgang mit Wissen und Information, nicht mit den entwickelten Leitideen zusammengehen. Mark Surman ${ }^{77}$, Mozilla Foundation, sprich von „Outsourcing von Ethik an Maschinen“ - er meint mit Maschinen KI-Programme. ${ }^{78} \mathrm{Ob}$ es sinnvoll oder möglich ist, ein Outsourcing der Informationsethik an das Urheberrecht, insbesondere an das Wissenschaftsurheberrecht, zu betreiben und sich bei der Politik dafür einzusetzen, ist eine Frage, die bezüglich des Sinnvollen bejaht, bezüglich des Möglichen nach den bisherigen Erfahrungen mit dem Urheberrecht offen bleiben muss. Ohnehin ist die These der Priorität von Ethik/Informationsethik nicht einfach zu verifizieren. Dafür braucht es eine längere Perspektive. „Siege“ der Ethik sind nicht rasch zu haben. Das schmälert die Pragmatik, die Handlungsrelevanz der These keineswegs.

\subsection{Wissen und Information. Wissensobjekte und Informationsobjekte}

Informationsethik ist ein Teilbereich der Informationswissenschaft.79 "Information“ nicht „Wissen“ steht dabei im Zentrum der fachlichen Diskussionen. Aber Information ist ohne Wissen nicht denkbar. Wissen ist die Voraussetzung dafür, dass etwas zu Information werden kann. Dafür soll in den folgenden beiden Unterabschnitten auf die spezielle informationswissenschaftliche Sicht auf Information bzw. auf die Beziehung zwischen Wissen und Information eingegangen werden. In 4.2 gehen wir auf Informationsethik speziell ein und in 4.3 auf die Begründung der in 3.3 bis 3.5 behandelten Leitideen durch den Commons-Ansatz der Institutionenökonomie. Dafür wird auch die Unterscheidung von Wissensobjekten und Informationsobjekten angewendet.

76 Ausgeführt z. B. in (Kuhlen 2014a) Interdependenzen zwischen Informationsethik und politischem Handeln; (Kuhlen 2014b) Copyright and information ethics - an insight; (Kuhlen 2010c) Ethical foundation of knowledge as a common; (Kuhlen 2004c) Informationsethik.

77 (Surman 2019) Simple Geschichten über KI sind eine Gefahr.

78 Vgl. die Arbeiten von Oliver Bendel zur Maschinenethik, zuletzt (Bendel 2019) Wozu brauchen wir die Maschinenethik?

79 Vgl. (Kuhlen 2004d) Artikel Informationsethik. 


\subsubsection{Wissen}

Mehr als eine Arbeitsdefinition für Wissen kann hier nicht angebracht werden. Die gesamte Philosophiegeschichte zu Wissen muss außen vor bleiben. Lediglich ein Satz zu Anfang des Artikels „Wissen“ 80 soll als Ausgang für eine Arbeitsdefinition verwendet werden:

In der Philosophie wird Wissen in erster Linie im Sinne bestimmter Fähigkeiten zur Rechtfertigung von Meinungen bzw. zur Erklärung von Tatsachen verstanden.

Wissen artikuliert sich in Vorstellungen oder Aussagen über Sachverhalte oder Objekte in der realen oder fiktiven Außenwelt. Diese Aussagen können empirisch gewonnen, theoretisch verifiziert oder aus internem Wissen abgeleitet sein. Wissen als Vorstellung ist zunächst eine kognitive Struktur in unserem Gehirn. Aussagen artikulieren sich weitgehend über natürliche oder künstliche bzw. formale Sprachen. Wissen als Vorstellung kann sich aber auch in anderen medialen Strukturen realisieren. Auch jede Form von Kunst realisiert Vorstellungen über Sachverhalte oder Objekte in der realen oder fiktiven Außenwelt. Hier geht es um Wissen in Bildung und Wissenschaft. Wissen als kognitive Struktur, solange es also in unseren Köpfen ist, gehört nur uns. Niemand kann unseren Gehirnen Wissen entnehmen (bislang jedenfalls nicht) und uns das nehmen, was wir für uns, sozusagen wortwörtlich als Eigen behalten wollen. Das ist aber nicht Eigentum, wie es vom Urheberrecht angesprochen wird. Das Eigene sozusagen im Kopf kann nicht von Dritten gebraucht, missbraucht, verknappt, enteignet, eingeschränkt und auch nicht vergütet werden. ${ }^{81}$ Tatsächlich ist Wissen nur dadurch urheberrechtlich relevant, als es in den verschiedenen medialen Formen von Werken repräsentiert ist. Allerdings stimmt der Satz, dass Wissen in unseren Köpfen nur uns gehört, dass Wissen in uns ein Eigenes und damit quasi unser Eigentum sei, in seiner Allgemeinheit wohl nicht. Vom ersten Augenblick unseres Lebens nehmen wir kontinuierlich Wissen auf, das andere geschaffen haben, auch über viele Generationen zurück. ${ }^{82}$ Man kann sich kein „neues“ Wissen vorstellen, welches nicht in irgendeiner Form auf bereits

80 (Wissen 2004) Artikel Wissen. In Historisches Wörterbuch der Philosophie, Spalte 855.

81 Diese exklusive Verfügung über das interne Eigene mag in Zukunft hinfällig werden, wenn es möglich sein sollte, die gehirninterne Kodierung dieses Wissens zu identifizieren und sozusagen den immateriellen Wissenstransfer möglich zu machen, ohne dass reale Wissensobjekte dabei beteiligt sind. Das ist derzeit noch mehr Dystopie als Utopie.

82 Selbst Goethe, der oft sozusagen als Kronzeuge für die Annahme eines individuellen kreativen Schöpfers von Werken angesehen und durch den das Konzept des geistigen Eigentums bestätigt wird, sah seine Werke eher als Produkt „eines Kollektivwesens“ an: „Alles, was ich gesehen, gehört und beobachtet, habe ich gesammelt und ausgenutzt. Meine Werke sind von unzähligen verschiedenen Individuen genährt worden, von Ignoranten und Weisen, Leuten von Geist und 
bestehendes Wissen Bezug nimmt bzw. in dieses integriert ist. Genaugenommen kann sich eigenes „neues“ Wissen nur darauf beziehen, dass es in der Kombination mit Wissen Anderer in einen neuen originellen Zusammenhang gestellt wurde. Dem trägt auch das Urheberrecht dadurch Rechnung, dass Schutzansprüche auch gelten, wenn aus dem sozusagen kombinatorischen, aus vielen Kontexten entstandenen Wissen ein Werk in der wahrnehmbaren Außenwelt entstanden ist. Kriterium ist weitgehend nur, dass dieses Werk so noch nicht vorhanden war. Was geschieht aber mit dem Wissen, wenn der Urheber des in einem Werk repräsentierten Wissens sich entschlossen hat, dieses Werk öffentlich und damit kommunizierbar zu machen? Auf diese Frage gibt es viele und auch viele berühmte und wunderbare Antworten. Hier eine besonders wunderbare Antwort von Thomas Jefferson von 1813:

If nature has made any one thing less susceptible than all others of exclusive property, it is the action of the thinking power called an idea, which an individual may exclusively possess as long as he keeps it to himself; but the moment it is divulged, it forces itself into the possession of every one, and the receiver cannot dispossess himself of it. Its peculiar character, too, is that no one possesses the less, because every other possesses the whole of it. He who receives an idea from me, receives instruction himself without lessening mine; as he who lights his taper at mine, receives light without darkening me. ${ }^{83}$

In dem Moment, wenn Wissen verbreitet (divulged), öffentlich zugänglich gemacht worden ist, will es (it forces itself), dass es von jedermann in Besitz genommen werden kann, ohne dass durch diese Besitznahme andere daran gehindert werden, es ebenfalls in Besitz zu nehmen. Auch durch das Urheberrecht sind Wissen, Ideen, Erkenntnisse, ... nicht geschützt. Jedermann kann Wissen frei nutzen. In diesem Sinne ist Wissen tatsächlich quasi vogelfrei. Wenn es allerdings in einer Version des Wissensautors repräsentiert und öffentlich gemacht wird, ist es geschützt. Aber dieses Wissen darf durch den Nutzer sozusagen paraphrasiert in einer neuen Version als Werk reklamiert werden. Dagegen hat das Urheberrecht keine Einwände und verlangt auch keine Referenz auf die erste Werks-/Autorenversion. In der Wissenschaft hingegen wird Letzteres verlangt, darin strenger als das Urheberrecht.

Wissen (in Werken) ist in mehrfacher Hinsicht nachhaltig. Es ist in vielfältigen Nutzungssituationen immer wieder verwendbar; es sichert den Transfer

\footnotetext{
Dummköpfen; die Kindheit, das reife und das Greisenalter, alle haben mir ihre Gedanken entgegengebracht, ihre ... Lebensansichten; ich habe oft geerntet, was andere gesät haben, mein Werk ist das eines Kollektivwesens, das den Namen Goethe trägt.“ Aus dem Briefwechsel Goethes mit Friedrich Soret, hrsg. Weimar 1905. Diesen Hinweis verdanke ich (Helfrich/Bollier 2019) Frei, fair und lebendig - Die Macht der Commons.

83 The Letters of Thomas Jefferson: No patents on ideas. To Isaac McPherson, Monticello, August 13, 1813 - https://bit.ly/35JDRvB.p
} 
der Aussagen früherer Generationen in Gegenwart und Zukunft; es nutzt sich durch Nutzung nicht ab, ganz im Gegenteil, je mehr es genutzt wird, umso mehr Nutzen stiftet es, und es ist damit nicht rivalisierend im Gebrauch. Meine Nutzung schließt die durch einen anderen nicht aus. Wissen entspricht damit - ohne dass wir uns diese Terminologie hier zu eigen machen wollen - dem ökonomischen Verständnis eines öffentlichen Guts. Für das Urheberrecht ist es, wie es auch schon das Jefferson-Zitat nahelegte, selbstverständlich, dass Wissen (Ideen, ...) frei nutzbar ist. Aber was nützt diese Freiheit, wenn der Zugriff auf das repräsentierte Wissen nicht vergleichbar frei ist? Nicht Wissen ist die Herausforderung, sondern der Zugriff darauf.

Fazit. Die Urheberrechtswissenschaft (vgl. 5.2) hat zwischen Werk und Werkstück unterschieden. Wir werden diese Unterscheidung zwischen dem immateriellen Charakter des Werks und der materiellen Struktur des Werkstücks in Kap. 6 kritisieren. Das Urheberrecht tut so, als ob das Werk als geistiges Konstrukt tatsächlich ein Objekt für sich ist und damit als Gut handelbar und durch das Gesetz regulierbar wird. Diese Terminologie - und damit die Implikationen für diese Unterscheidung wird hier nicht verwendet. Vielmehr wird das über eine Repräsentationsform wahrnehmbare Wissen als Wissensobjekt bezeichnet. Je nach Gegenstandsbereich wird die Repräsentationsform eine andere sein. In der Wissenschaft ist es überwiegend die Sprache - nicht nur die natürliche, als Fachsprache dem jeweiligen Fachgebiet angepasste Sprache, sondern auch formale Sprachen. Die Leistung, ein Wissensobjekt zu erstellen, besteht nicht nur in der semantischen Repräsentation, sondern auch in der syntaktischen strukturierten Linearisierung und Textualisierung der verschiedenen Wissensfragmente. Das Wissensobjekt hat vor einer Veröffentlichung nur das Potenzial, kommunizierbar und nutzbar zu sein. Erst dadurch, dass sich der Wissenschaftler entscheidet - und nur er hat nach dem Urheberrecht dieses Entscheidungsrecht -, sein Wissensobjekt öffentlich zu machen, wird er zum Urheber im rechtlichen Sinne (auch wenn das Urheberrecht darauf beharrt, dass auch das Wissensobjekt in der Schublade urheberrechtlich geschützt ist). Erst dann, also durch die Veröffentlichung, ergibt sich für die (Fach-)Öffentlichkeit die Möglichkeit, aus dem Wissensobjekt Information abzuleiten. Die Veröffentlichung macht Wissensobjekte zu Informationsobjekten.

\subsubsection{Information}

Mit dem Öffentlichmachen der Wissensobjekte kommt Information ins Spiel. Informationen referenzieren auf repräsentiertes Wissen, auf Wissensobjekte, wie wir sie oben genannt haben; aber sie entfalten diese Bedeutung nur mit Referenz auf 
die aktuelle Benutzungssituation. Information hat durch den Bezug auf Wissen eine semantische Dimension, durch den Bezug auf das Handeln bzw. der Folgen der Verwendung von Wissen eine pragmatische Dimension. Informationen bedeuten also etwas, aber - und das macht das pragmatische Grundverständnis aus - sie existieren nicht losgelöst von ihrer Nutzung und von den Folgen, die deren Nutzung nach sich ziehen. Von Information kann man nur im aktuellen Kontext ihrer Verwendung sprechen, unter Berücksichtigung der verschiedenen Rahmenbedingungen ihrer Benutzung. ${ }^{84}$ Nimmt man es genau, kann man von Information nur sprechen, wenn das dabei referenzierte Wissen für den Nutzer neu ist. Was man schon weiß, ist keine Information. Nützlich kann eine gewisse Redundanz dennoch sein, wenn dadurch das bislang nur vage Gewusste in der Gewissheitsskala etwas nach oben rückt. Für dieses pragmatische Verständnis von Information - als aktiv gewordenes Wissen ${ }^{85}$ - steht die Formel Information ist Wissen in Aktion (Kuhlen 2013c). Entsprechend diesem pragmatischen Verständnis referenziert Information auf das Wissen, das in aktuellen Handlungssituationen benötigt wird, z. B. zur Problemlösung oder um Entscheidungen treffen zu können, aber das der aktuell Handelnde nicht selbst hat.

Information als handlungsrelevantes Wissen macht keine Aussage über den Wahrheitswert. Information kann auch aus Meinungen oder sogar aus falschen Aussagen entstehen. Falsche Aussagen, also Wissen, das im traditionellen Verständnis kein Wissen ist, können Konsequenzen für aktuelles Handeln haben sei es, dass Personen deren Wahrheitswert nicht einschätzen können und sie für wahr halten, oder sei es, dass Personen, aus welchen Gründen auch immer, bewusst falsche Aussagen in die Welt setzen, in der Erwartung dass diese als

\footnotetext{
$84 \mathrm{Zu}$ den Rahmenbedingungen gehören z. B. kognitive Faktoren wie der bisherige Wissensstand des Informationssuchenden, seine Gedächtnisleistung, seine Informationsverarbeitungskapazität und situative, soziale Faktoren, z. B. die Dringlichkeit der Nutzung, der Verwendungszweck, die Verfügbarkeit der technischen Ressourcen für Suche, der organisationelle Hintergrund, die allgemeine Informationskultur der aktuellen Umgebung und auch die (urheber)rechtlichen Vorgaben für die Nutzung von Information. Verschärft wird die Anforderung an Information nicht nur dadurch, dass sie relevant, also einschlägig für die aktuelle Situation, sondern auch noch in ihrem Wissensgehalt neu für den Aufnehmenden sein muss. Etwas, was man schon weiß, ist keine Information.

85 Auch dieser Vorschlag ist im Searle'schen Sinne ein deklarativer Sprechakt, eine semantische und pragmatische Festschreibung von „Information“, die aber nur zu einer allgemeinen informationswissenschaftlichen (oder sogar allgemeinen) Institutionalisierung führen kann, wenn eine relevante Mehrheit der davon betroffenen Akteure sich dieser Deklaration anschließen kann. Derzeit spricht vieles dafür, dass (auch weltweit) die aktiven Informationswissenschaftler ihre Arbeit auf diesem pragmatischen Verständnis von Information gründen und Information unter den Aspekten der Nutzung, des Nutzens und der Wirkung untersuchen.
} 
Information aufgegriffen und zu den von den Falschmeldern erwünschten Handlungen veranlasst werden. ${ }^{86}$ Informationskompetenz, die Fähigkeit, Information mit niedrigem von solchen mit hohem Wahrheitswert zu unterscheiden, erkennen $\mathrm{zu}$ erkennen, was manipulative und was begründete Information ist, wird somit zu einem entscheidenden Bildungsziel, vor allem in elektronischen Umgebungen. Informationskompetenz ist das, was in der Philosophie bestimmende Urteilskraft genannt wird, das Neue dem bestehenden gesicherten Wissen zuordnen zu können bzw. es aus guten Gründen ablehnen zu können.

Die semantische und pragmatische Dimension von Information liegt nicht nur der Informationsethik zugrunde (s. unten), sondern ist auch aus urheberrechtlicher Sicht einschlägig. Wir haben oben in dem Abschnitt über Wissen (4.1.1) das von einem Autor in einer „Sprache“ repräsentierte, linearisierte und für eine Veröffentlichung vorgesehene Wissen als Wissensobjekt bezeichnet. Diese Wissensobjekte müssen veröffentlicht sein, damit sie wahrgenommen und genutzt werden können. Das kann auf vielfältige Weise geschehen. Der traditionelle Weg, dieses Ziel zu erreichen, geschieht über eine Veröffentlichung durch einen Verlag. Auf die urheberrechtliche Übertragung der Autorenrechte an den Wissensobjekten als Nutzungsrechte der Verleger wird in 5.5 eingegangen, auf deren Problematik in 6.6. Hier geht es um die pragmatische Dimension der Veröffentlichung. Der Zweck der Veröffentlichung ist aus Verlagssicht, einen Gewinn damit zu erzielen. Der generelle Zweck der Veröffentlichung in Bildung und Wissenschaft liegt aber darin, dass das in ihr enthaltene Wissen genutzt werden kann. Anders gesagt: Wissensobjekte werden als Informationsobjekte öffentlich gemacht, damit aus ihnen Information entsprechend ihres semantischen Gehalts und ihrer pragmatischen Relevanz abgeleitet werden kann.

Fazit. Wir unterscheiden Wissensobjekte als Ergebnis der Arbeit der Autoren und Informationsobjekte als Angebote zur Nutzung auf den Informationsmärkten. Entsprechend der Unterscheidung von kommerziellen proprietären und offenen freien Informationsmärkten können diese Informationsobjekte von den Autoren selbst erstellt und zur Nutzung freigegeben werden, z. B. indem sie ohne einen Verlag von den Autoren auf ihre Website gestellt werden. Die Wissensobjekte können aber auch von Organisationen aus der Wissenschaft selbst, Bibliotheken, Fachverbände etc., als Informationsobjekte zur Nutzung öffentlich gemacht werden.

86 Damit ist dieses hier skizzierte Verständnis von Information auch relevant für die derzeitige Diskussion zu Fake News im Internet. Vgl. (Hauff-Hartig 2018) greift dazu die These in (Kuhlen 2013c) auf: „Von Wahrheit ist dabei nicht die Rede. Auch eine Lüge kann eine Information sein.“ Information gilt daher auch „zwangsläufig für Phänomene wie Desinformation, Fehlinformation und Falschinformation.“ 
Oder eben von kommerziellen Unternehmen wie Verlagen. In 4.3 werden wir die Umwandlung von Wissensobjekten in unterschiedlich institutionell realisierte Informationsobjekte weiter diskutieren mit Rückgriff auf die Unterscheidung von Common Pool Resources (CPR) und Commons.

\subsection{Zur Begründung der Informationsethik}

Die Bezeichnung „Informationsethik“ (information ethics) hatte sich international in den drei UNESCO-INFOethics-Konferenzen (1997, 1990 und 2000) durchgesetzt. ${ }^{87}$ Informationsethik ist nicht etwas grundlegend Neues gegenüber der langen philosophischen Tradition von Ethik allgemein. Auch in der Informationsethik geht es um Werte wie Freiheit, Gerechtigkeit, Gleichheit, Vertrauen, ... oder um (Grund-) Rechte wie Würde, Eigentum, Meinungs-, Religions-, Informations-, Presse-, Wissenschaftsfreiheit, ... Informationsethik im hier vertretenen Sinne gründet sich nicht auf einem quasi naturrechtlichen Verständnis solcher Werte und Rechte, genauso wie hier nicht von einer naturrechtlichen Begründung des Urheberrechts bzw. des ihm zugrundeliegenden Eigentumsverständnisses ausgegangen wird. Vielmehr wird Informationsethik hier auf eine philosophische, auf Aristoteles zurückgehende Tradition verpflichtet. ${ }^{88}$

Für Aristoteles war es das tatsächliche Leben in dem Raum der Athenischen Polis, welches die Werte und Verhaltensformen der Menschen bestimmt. Sie sind also nicht wie in Platonischer Tradition absolut - sei es metaphysisch, religiös oder naturrechtlich - zu begründen, sondern binden sich zurück an Gewohnheiten, die sich zu Verhaltensformen, Traditionen sowie Normen und Wertvorstellungen verdichten, wenn sie von den Menschen, die in vergleichbaren Räumen leben, allgemein akzeptiert werden. Die Gesamtheit der Verhaltensformen, Normen und Wertvorstellungen, die unser Handeln bestimmen, kann als Moral bezeichnet werden. Moralische Vorstellungen fallen nicht vom Himmel (auch wenn sie in großen Teilen der Welt weiter aus allgemeinen ethischen bzw. bzw. fundamentalen religiösen Vorgaben direkt abgeleitet werden), sondern entwickeln sich in den Räumen, den Umgebungen, in denen die Menschen leben und handeln. Sie sind nicht stabil, sondern verändern sich mit den Rahmenbedingungen, die diese Räume, die Umgebungen strukturieren.

Die Räume in der Gegenwart, in denen sich diese Entwicklungen vollziehen, sind die elektronischen Räume des Internets. Das Internet ist aus der Sicht der

87 Zuweilen wird auch von „Wissensethik“ gesprochen; vgl. (von Guretzky o. J.) Wissensethik.

88 Vgl. (Ritter 1972) Artikel Ethik. 
Informationsethik der Raum, das Ensemble der intellektuellen Lebenswelten, in dem beim Umgang mit Wissen und Information neue Verhaltensformen, neue Normen/Werte bzw. ein neues Verständnis von bestehenden Werten entstehen. In ihnen „bewegen” wir uns unabhängig von räumlichen und zeitlichen Beschränkungen, und aus ihnen reproduzieren wir immer mehr unser intellektuelles Leben. ${ }^{89}$ Die elektronischen Umgebungen bestimmen in beträchtlichem Ausmaß unsere Sicht von Welt. Wir erfahren Welt zu großen Teilen nicht mehr direkt aus dem, was wir real erleben, sondern aus der telemediatisierten Welt und zwar in weitaus stärkerem Umfang, als es bislang durch die Vermittlung traditioneller Medienwelten (Zeitungen, Rundfunk, Fernsehen) der Fall war. So wenig wie moralische Vorstellungen und Verhaltensformen quasi vom Himmel fallen, so wenig sind sie unabhängig vom Stand der Technologien oder von den Organisationsformen der Märkte und ebenso nicht unabhängig vom Stand oder den Plänen politischer Regulierung. Der theoretische Ansatz für Moral besagte ja gerade, dass sich diese in den sich historisch wandelnden „Räumen“ entwickelt, in den die Menschen leben und handeln. Diese Räume werden beeinflusst vom Stand der Technologien, von den Formen wirtschaftlichen Handelns und von den Ausprägungen des politischadministrativen Systems, und damit wirken sie auch auf „Moral“ ein. Entsprechend gilt genauso, dass Technologien und Märkte sich nicht naturgesetzlich autonom entwickeln, sondern sich wechselseitig beeinflussen, aber auch von dem moralischen „Empfinden“ der Menschen und den gesetzlichen und administrativen Vorgaben der Politik mitbestimmt werden.

Lawrence Lessig ${ }^{90}$ hat in seinem Code-Buch für diese Zusammenhänge eine Vorgabe gegeben, indem er von den vier constraints spricht, die auf komplexe Sachverhalte in Gesellschaften einwirken: norms, law, market, architecture. Hier werden diese constraints mit Blick auf den hier zu behandelnden Gegenstand dadurch konkretisiert, dass norms durch Informationsethik, law durch Urheberrecht, market durch kommerzielle und offene Informationsmärkte und architecture durch Informations- und Kommunikationstechnologie ersetzt werden. Diese Lessig'schen constraints werden hier als Regulierungsinstanzen angesprochen. Für diese gibt es keine klaren Hierarchien oder Dominanten. Plausibler ist es, den Zusammenhang der vier Regulierungsformen durch eine umfassende Interdependenz zu bestimmen.

Die anfangs aufgestellte Prioritätsthese zugunsten der Informationsethik ist kein Widerspruch zu dieser Interdependenz der verschiedenen Regulierungsinstanzen. Informationsethik muss sich nicht an einem rechtlich vorgegebenen Rahmen

89 Vgl. den entsprechenden Ansatz in (Capurro 2003) Infomationsethik. Eine Standortbestimmung; (Hausmanninger/Capurro 2002) Netzethik. Grundlegungsfragen der Internetethik. 90 (Lessig 1999/2006) - 1999, S. 86ff bzw. 2006, S. 120 ff. 
orientieren. Informationsethische Argumente müssen nicht kompatibel mit der bestehenden Rechtsordnung sein. Das Ziel informationsethischer Diskurse besteht nicht darin, zum Einhalten urheberrechtlicher Bestimmungen zu erziehen. Das ist hier nicht mit Informationsethik gemeint. Informationsethik macht eine Differenz zum Bestehenden. Das muss so sein. Das moralische Bewusstsein entwickelt sich schneller, als es das Recht kann - treffender gesagt: als es das Recht darf. Das Recht muss für Sicherheit sorgen. Informationsethik muss Unruhe als Bedingung für Weiterentwicklung auch des Rechts schaffen. ${ }^{91}$

Angesichts der Präferenz des Rechts für Stabilität ist in der Tat zu erwarten, dass das Ergebnis informationsethischer Diskurse häufig eine Differenz informationsethischer Erwartungen zu den bestehenden rechtlichen Normen aufscheinen lässt. Soll die anfangs formulierte Prioritätsthese Bestand haben, so kann sich Informationsethik nicht mit der analytischen Deskription und der daraus folgenden Kritik begnügen. Das Ziel der Informationsethik besteht vielmehr darin, die noch diffus sich entwickelnden und zuweilen auch sich widersprechenden moralischen Vorstellungen (in der Gesamtheit: die Moral) für den Umgang mit Wissen und Information zu stabilisieren und sie zu Leitideen zusammenzufassen und diese durch operative Handlungsanweisungen zu konkretisieren.

Leitideen spiegeln nicht das Sein wider, sondern leuchten über das Sollen die Bedingungen für ein neues Sein aus. Leitideen können zu jedem gegebenen Zeitpunkt nicht nur entwicklungsoffen, sondern können unter einer gesamtgesellschaftlichen Sicht auch nicht einheitlich sein. Neue normative Vorstellungen und Verhaltensformen entwickeln sich keineswegs zeitgleich in den verschiedenen gesellschaftlichen Gruppierungen und werden auch keineswegs von allen Gruppierungen gleichermaßen geteilt. Wir haben schon in 3.3 darauf hingewiesen, dass das in Leitideen verdichtete moralische Verhalten von Personen und Akteursgruppen, die durch analoge Umgebungen primär geprägt wurden, ein anderes ist als von Personen und Institutionen, denen die elektronischen Umgebungen die primären Lebenswelten geworden sind. Da ist eine besondere Herausforderung für die Gestaltung des Urheberrechts. Oft genug (und oft viel zu lange) ist es den herkömmlichen Leitideen aus der analogen Welt gefolgt (Beispiele dafür in Teil II). Es ist Aufgabe der Informationsethik, über wertebasierte Diskurse zu ermitteln, welches die gegenwärtig dominierenden Leitideen für den Umgang mit Wissen und Information sind. Es ist die Aufgabe des Urheberrechts, die in den Leitideen entwickelten konkreten Handlungsanweisungen in rechtlich verbindliche Normen umzusetzen. Dadurch können und sollen frühere Leitideen

91 Entsprechend der auf die Gesamtheit der Moderne gerichteten Analyse von (Konersmann 2015) Die Unruhe der Welt. 
nicht einfach sozusagen über Bord geworfen werden. Aber bloße Kompromisse mit gleicher Gewichtung der heterogenen Leitideen schaffen kein zeitgemäßes und zukunftsoffenes Urheberrecht. Wir plädieren hier für informationsethisch begründete Prioritäten.

Fazit. Informationsethik kann unter einer dreifachen Perspektive bestimmt werden - deskriptiv, normativ, konstruktiv: (1) Informationsethik beschreibt aus Produzenten-/Verwerter- und Nutzersicht, welche Wertvorstellungen und normative Verhaltensformen (welche Moral) in den Lebenswelten, heute immer mehr in den elektronischen Räumen des Internets für den Umgang mit Wissen und Information bestehen bzw. sich entwickeln. (2) Informationsethik unternimmt es, aus den Wertvorstellungen und normativen Verhaltensformen - unter Abwägung der verschiedenen Interessen der beteiligten Akteursgruppen und unter Berücksichtigung von ethischen Theorien allgemein - Leitideen für den Umgang mit Wissen und Information abzuleiten. (3) Informationsethik entwickelt Vorschläge, durch welche Maßnahmen in welchen Objektbereichen und unter Berücksichtigung der verschiedenen Regulierungsinstanzen die Leitideen in die Praxis umgesetzt werden können. Wir werden im folgenden Abschnitt herausarbeiten, dass aus Sicht der Informationsethik die zentrale Leitidee für den Umgang mit Wissen und Information (vgl. 3.3-3.5) die nachhaltige commons-basierte Nutzungsfreiheit ist.

\subsection{Zur Begründung von Leitideen durch institutionalisierte Commons}

Spätesten seit dem grundlegenden Band von (Hess/Ostrom 2007) hat sich das Verständnis durchgesetzt, dass auch Wissen, also nicht nur natürliche Ressourcen, als Commons, als immaterielle Commons zu begreifen sind. Entsprechend wollen wir den allgemeinen Begriff der Ökologie auf Wissensökologie erweitern. ${ }^{92}$ So wie die allgemeine Ökonomie sich immer mehr Grundsätze der Ökologie zu eigen macht, so deutet Einiges darauf hin, dass auch die traditionelle Wissens-/Informationsökonomie sich veranlasst sieht, ihre Geschäftsmodelle nach wissensökologischen Prinzipien zu organisieren. Ökologie der natürlichen und Ökologie der immateriellen Ressourcen scheinen zunächst im Widerspruch zueinander zu stehen. Die Nachhaltigkeit der natürlichen Ressourcen, einschließlich des

92 Vgl. (Kuhlen 2004f) Artikel Wissensökologie; (Kuhlen 2004e) Nachhaltigkeit muss nicht Verknappung bedeuten - in Richtung Wissensökologie; (Kuhlen 2012b) Wissensökonomie und Wissensökologie zusammen denken. 
Anspruchs späterer Generationen auf deren Verfügbarkeit, soll vor allem dadurch gesichert werden, dass sie vor Übernutzung geschützt und daher auch verknappt werden müssen. Das gilt für Wissen nicht. Je mehr es genutzt wird, desto größer der Nutzen. Nachhaltigkeit bedeutet entsprechend hier gerade nicht Schutz vor Übernutzung, sondern Schutz vor Unternutzung. Unternutzung mag nicht das primäre Ziel des Urheberrechts sein. Aber für Bildung und Wissenschaft bewirkt das Urheberrecht genau die Unternutzung durch Verknappung des Zugriffs und der Nutzung. Entscheidend zur Verknappung hat beigetragen, dass das Urheberrecht bestimmende Konzept des geistigen Eigentums des Urhebers auch auf die kommerzielle Verwertung mit entsprechenden Vergütungsansprüchen übertragen wird.

Für Wissensökologie und damit für immaterielle Ressourcen gilt die gleiche ethische Begründung wie für die von materiellen Ressourcen. So wie Wasser unabdingbar ist für die menschliche Existenz, so ist Wissen unabdingbar für soziale und individuelle Entwicklung. Wissen ist sozusagen zu Wasser äquivalent, oder, besser im Englischen auszudrücken als im Deutschen: „Knowledge is the water of the mind“. ${ }^{93}$ Nach (Hess/Ostrom 2007) ist Wissen das Ergebnis der menschlichen Entwicklung und das Ensemble der intellektuellen Aktivitäten, die in einer medialen Form öffentlich zugänglich gemacht worden sind. Wissen ist damit Teil des gemeinsamen menschlichen Erbes. Wissen ist vom Prinzip ein Gemeinschaftsgut, das der Gemeinfreiheit unterliegt. Das anerkennt auch das Urheberrecht. Wissen kann von jedermann ohne Einschränkung genutzt werden, wenn es denn bekannt gemacht werden kann. Wissen ist also nicht für sich zugreifbar oder nutzbar, sondern nur wenn es in einer medialen Gestalt repräsentiert ist und dieses Ergebnis öffentlich zugänglich gemacht. Nach dem Ostrom'schen Verständnis von Institutionenökonomie (vgl. FN 96) entstehen Commons erst dadurch, dass die sie begründenden CPR (wie Wasser, aber eben auch Wissen) durch soziale Konstrukte wie Konsens, Vereinbarungen, Verpflichtungen, Verträge, Regeln, Gesetze, aber auch durch Kontrollmechanismen oder Sanktionen nutzbar werden. Commons wie Wissen und Information sind keine ontologisch stabilen Entitäten. Commons entstehen nicht zwangsläufig, sondern sind in der Form ihrer Realisierung durchaus kontingent. Commons werden erst im Prozess des Commoning gebildet, ${ }^{94}$ durch Initiativen der jeweiligen Akteure, welche dafür sorgen, dass Commons auf nachhaltige Weise genutzt werden können. Commons sind, darin vergleichbar mit der Bestimmung von „Information”, ein soziales Phänomen. Sie existieren nicht für sich, sondern nur im Kontext ihrer Anwendung durch die daran interessierten und betroffenen

93 (Kuhlen 2012c) Knowledge is the water of the mind.

94 (Helfrich/Bollier 2019) Frei, fair und lebendig - Die Macht der Commons. 
Akteure. Sie werden, um eine Formulierung von Berger/Luckmann aufzugreifen, sozial konstruiert. ${ }^{95}$ Der Prozess des Commoning, die Initiativen der Akteure, wird zwar in der Commons-Literatur an Hand von vielen Beispielen ${ }^{96} \mathrm{zu}^{\mathrm{Rech}}$ und unabdingbar als autonom, selbstorganisiert und selbstbestimmt beschrieben, aber die Prozesse des Commoning sind nicht unabhängig

- von den technologischen Rahmenbedingungen für Produktion, Aufbereitung, Verteilung und Nutzen von Wissen und Information,

- von den entwickelten und sich neu abzeichnenden Geschäftsmodelle auf den Informationsmärkten,

- $\quad$ von dem in der Gesetzgebung sich darstellendem politischen Willen und -

- unter Beachtung der Prioritätsthese - von der entwickelten öffentlichen Moral als dem Ensemble der Werte/Prinzipien/Normen und den darauf beruhenden Kommunikationsformen.

Dies sind die in 4.2 angesprochenen Regulierungsinstanzen, deren Beziehung untereinander multidirektional sind. Technologie beeinflusst moralische Einstellungen, aber diese, zusammen mit anderen Faktoren wie politischer Wille und ökonomisches Interesse, steuern auch die Wahl aus den verfügbaren Technologien. So kann Technologie (hier als Software) zum Einsatz von Digital Rights Management (DRM) und Filtern ${ }^{97}$ zum Schutz kommerzieller Marktinteressen und zur Durchsetzung von urheberrechtlichen Regelungen führen. Sie kann aber auch die Entwicklung von offenen Informationsmärkten in Open-Access- bzw. Open-Science-Modellen für das freie Teilen von Wissen und Information begünstigen. Vor allem durch die rasche Technologieentwicklung ist die Umsetzung von CPR in Commons in hohem Maße entwicklungsoffen/kontingent. Produktion, Verteilung und Nutzung

95 (Berger/Luckmann 1966/1969) Die gesellschaftliche Konstruktion der Wirklichkeit.

96 (Hess/Ostrom 2007) Understanding knowledge as a commons; (Ostrom et al. 2008) Rules, games, \& common-pool resources 2008); (Helfrich et al. 2009) Gemeingüter - Wohlstand durch Teilen; (Helfrich/HBS 2012) Commons. Für eine neue Politik jenseits von Markt und Staat; (Bollier/ Helfrich 2012) The wealth of the commons; (Helfrich/Bollier 2019) Frei, fair und lebendig - Die Macht der Commons.

97 In der Form von „Upload-Filtern“ durch Art. 17 in der EU-Urheberrechtsreform von 2019. Das Wort „Upload-Filter“ selbst kommt in EU-DSM-RL2019, Art. 17 „Nutzung geschützter Inhalte durch Diensteanbieter für das Teilen von Online-Inhalten“ nicht vor - intentional aber wohl doch. Inwieweit solche Filter dennoch verbindlich von den Mitgliedsländern eingesetzt werden, werden die nächsten Jahre zeigen. Wandtke/Hauck sehen in Art. 17 „einen Paradigmenwechsel bei der Durchsetzung des Urheberrechts. Die Rechteinhaber werden gestärkt, sie müssen zukünftig insbesondere angemessen an den Einnahmen der Diensteanbieter beteiligt werden. “ (Wandtke/ Hauck 2019) Art. 17 DSM-Richtlinie. Ob dafür Upload-Filter ein geeignetes Instrument sein kann bzw. sein soll, kann durchaus bezweifelt werden. 
von Wissen und Information waren zu einer Zeit, als Wissen durch Schreiben auf Pergament oder vorher auf Tierhäuten als Informationsobjekte sichtbar gemacht wurden, gänzlich andere gegenüber den Möglichkeiten, die sich durch Gutenbergs Entwicklung des Drucks mit beweglichen Buchstaben ergeben haben. Diese sind heute in den elektronischen Umgebungen des Internets wiederum gänzlich andere. Technologische Veränderungen vollziehen sich aber auch laufend in „kleineren“ Veränderungen als nur durch große, wie eben angedeutete Paradigmenwechsel, z. B. durch

- die nicht-lineare Wissensorganisation, wie sie durch die Hypertextmethodologie möglich wurde,

- die breite Verfügbarkeit von multimedialen Repräsentationsformen,

- die Produktion von Wissen durch Anwendung von TDM-Algorithmen über große Datenmangen,

- durch KI-basierte automatisierte Lernverfahren zur Erweiterung und Absicherung bestehendes Wissen

- die enger werdende Verflechtung von Wissensproduzenten und Wissensnutzern (Stichwort: user-generated content),

- die intensivierte weltweite Kommunikation über soziale Netzwerke, durch die auch in der Wissenschaft ganz neue Diskurse und damit auch neue Wissensobjekte entstehen, die ohne weitere Aufbereitung und Vermittlung durch Dritte sofort zu nutzbaren Informationsobjekten werden.

All dies hat Einfluss auf die zur Anwendung kommenden Institutionalisierungsverfahren (der Transformation von CPR in Commons), sowohl mit Bezug auf die Prinzipien/Werte als auch auf die oben beschriebenen sozialen Konstrukte wie Vereinbarungen, Prozeduren/Regeln, etc. Technologische Rahmenbedingungen haben zudem Regulierungskonsequenzen, z. B. wer für was Eigentumsrechte reklamiert, über welche Geschäftsmodelle der Anspruch auf Verwertung behauptet wird. All das hat Konsequenzen für Zugriffs- und Nutzungsformen, für Verknappung oder Offenheit von Wissen und Information.

Bei der Diskussion der Leitideen wurde angemerkt, dass Konflikte häufig dadurch entstehen, dass unterschiedliche Leitideen aufeinanderprallen. Das sind zum einen Leitideen, die sich in einem früheren Umfeld entwickelt haben und die sich auf bestimmte Werte bzw. zeitabhängige Interpretationen von Werten stützen, und zum anderen Leitideen, die sich in der Gegenwart in einem weitgehend anderen Umfeld mit neuen Werten bzw. mit einem neuen Verständnis bestehender Werte orientieren. Ein Beispiel dafür ist der Konflikt zwischen der etwa 100 Jahre alten Leitidee für den Verkehr, nämlich dass jeder die Möglichkeit haben soll, seine Mobilität durch die individuelle Verfügung über ein Automobil zu sichern, und der gegenwärtig sich entwickelnden Leitidee, dass Mobilität durch überwiegend 
durch ein öffentliche Verkehrssystem gesichert werden soll. Die Ablösung der alten Leitidee durch die eine begründet sich dadurch, dass die alte Leitidee objektiv dysfunktional geworden ist: klimaschädlicher Ausstoß von CO2, großer Verbrauch natürlicher Ressourcen, dominierende Überlastung öffentlicher Räume vor allem in Städten. Diese objektive Dysfunktionalität hindert derzeit aber die Mehrheit der Bevölkerung nicht daran, der alten Leitidee zu folgen, obgleich diese gleichzeitig die neue Leitidee im Prinzip für richtig hält. Widersprüche zwischen Verhalten und Überzeugung lösen sich nicht schnell auf.

Die Geschichte (bis in die Gegenwart) hat gezeigt, dass durchaus auch private kommerzielle Verwertungsmodelle z. B. für die Ressource Wald für einen nachhaltigen und sozial angemessenen sorgen können, während für andere Ressourcen wie Luft oder auch Wasser eine nachhaltige und sozial verträgliche Sicherung wohl kaum anders als durch staatliche bzw. überstaatliche Regulierung erreicht werden kann. Aber Nachhaltigkeit kann (und wird zunehmend) auch durch das Commoning der von einer Ressource betroffenen Akteure erreicht. Das kann auch für die CPR Wissen angewendet werden. Auch für Wissen und Information war über viele Jahrhunderte das Öffentlichmachen von Wissensobjekten über entsprechende Informationsobjekte und die Sicherung des Zugriffs auf diese und damit die Nutzung von Wissen durch das Zusammenspiel von kommerzieller Verlagswirtschaft und öffentlich finanzierten Bibliotheken gesichert.

In 14.11 greifen wir den Ostrom'schen Ansatz der Institutionenökonomie noch einmal auf, um die prinzipielle Kompatibilität der beiden Informationsmärkte, der kommerziellen und der offenen Informationsmärkte, zu erklären. Kompatibel sind beide Märkte allerdings nur, wenn sich das kommerzielle Verknappungsmodell in ein offenes Nutzungsmodell transformiert. Gelingt diese Transformation, wie es sich in der Gegenwart andeutet, dann sollten die kommerziellen Akteure auf den wissenschaftlichen Informationsmärkten weiter aktiv präsent bleiben können. Die Transformation in Richtung Nutzungsfreiheit kann aber auch ganz anders verlaufen, nämlich dadurch, dass es nicht mehr die kommerziellen Akteure sind, die diese realisieren, sondern dass die freien offenen Publikationsmodelle aus der Wissenschaft selbst organisiert werden. Das wäre dann eine andere Form der Institutionalisierung der Bereitstellung von Wissen und Information. Auch das wird in Kap. 14 diskutiert. Beide Modelle sollten Auswirkungen auf die Gestaltung des Urheberrechts haben.

Fazit. Als Ergebnis dieses Kapitels kann festgehalten werden, dass in der Öffentlichkeit eine Leitidee sich entwickelt, nach der Wissen und damit auch die entsprechenden Informationsobjekte tatsächlich frei verfügbare Gemeingüter (Commons) sein sollen. Konkret besteht offensichtlich ein Konsens in der Öffentlichkeit darüber, dass speziell das mit öffentlichen Mitteln erzeugte Wissen 
grundsätzlich frei sein sollte. Der freie Zugriff und die freie Nutzung von Wissen repräsentierenden Informationsobjekten sollten der Default-Wert sein und die kommerzielle Verwertung die Ausnahme. Es verstärken sich die Hinweise, dass die Leitidee der freien Nutzung des mit öffentlichen Mitteln erzeugtem Wissens auch von der Politik aufgegriffen wird und dass die Informationsmärkte darauf mit neuen Modellen reagieren. Die Verträglichkeit bzw. Einheit von Ökonomie und Ökologie, der ökonomischen und ökologischen Prinzipien ist seit vielen Jahren für Wirtschaft, aber auch für die Politik ein Selbstverständlich geworden. Das sollte auch für Wissensökonomie und Wissensökologie gelten. So wie z. B. Klimaschutz die Chancen auf wirtschaftliches Wachstum und öffentliche Mobilität ganz neue Wirtschaftszweige entstehen lässt, so könnte auch der nachhaltige Umgang mit Wissen und Information der Motor für die Wissens- und Informationsindustrie sein. Nachhaltiger Umgang bedeutet hier gerade nicht Verknappung und Beschränkung der Nutzung wie bei den natürlichen Ressourcen, sondern freier Zugang und freie Nutzung zur Vermeidung von Wissensunternutzung. Je mehr Wissen genutzt, umso größer der wissenschaftliche, aber auch der allgemeine gesellschaftliche Nutzen. 